\title{
Some Properties of Solutions to Multiterm Fractional Boundary Value Problems with $p$-Laplacian Operator
}

\author{
KumSong Jong $\mathbb{D}^{1},{ }^{1}$ HuiChol Choi, ${ }^{1}$ KyongJun Jang, ${ }^{1}$ SongGuk Jong, ${ }^{1}$ KyongSon Jon, ${ }^{1}$ \\ and $\mathrm{Ok} \mathbf{R i}^{2}$
}

${ }^{1}$ Faculty of Mathematics, Kim Il Sung University, Pyongyang, Democratic People's Republic of Korea

${ }^{2}$ Kumsong Middle School No.2, Pyongyang, Democratic People's Republic of Korea

Correspondence should be addressed to KumSong Jong; ksjong1016@163.com

Received 13 July 2020; Revised 23 March 2021; Accepted 24 March 2021; Published 12 April 2021

Academic Editor: Richard I. Avery

Copyright ( 2021 KumSong Jong et al. This is an open access article distributed under the Creative Commons Attribution License, which permits unrestricted use, distribution, and reproduction in any medium, provided the original work is properly cited.

\begin{abstract}
In this paper, we study some properties of positive solutions to a class of multipoint boundary value problems for nonlinear multiterm fractional differential equations with $p$-Laplacian operator. Using the Banach contraction mapping principle, the existence, the uniqueness, the positivity, and the continuous dependency on $m$-point boundary conditions of the solutions to the given problem are investigated. Also, two examples are presented to demonstrate our main results.
\end{abstract}

\section{Introduction}

This paper deals with a class of multipoint boundary value problems for nonlinear multiterm fractional differential equations with $p$-Laplacian operator:

$$
\left\{\begin{array}{l}
D_{0+}^{\beta}\left(\varphi_{p}\left(D_{0+}^{\alpha} x(t)\right)\right)=f\left(t, x(t), D_{0+}^{\gamma} x(t)\right), 0<t<1, \\
D_{0+}^{\gamma} x(0)=0, D_{0+}^{\delta} x(1)=\sum_{i=1}^{m-2} \xi_{i} D_{0+}^{\delta} x\left(\eta_{i}\right), \\
D_{0+}^{\alpha} x(0)=0, \varphi_{p}\left(D_{0+}^{\alpha} x(1)\right)=\sum_{i=1}^{m-2} \zeta_{i} \varphi_{p}\left(D_{0+}^{\alpha} x\left(\eta_{i}\right)\right),
\end{array}\right.
$$

where $\quad 1<\alpha, \beta \leq 2,0<\gamma, \delta<1,0<\xi_{i}, \eta_{i}, \zeta_{i}<1, i=1,2, \cdots$, $m-2$ and $D_{0+}^{\alpha}, D_{0+}^{\beta}, D_{0+}^{\gamma}, D_{0+}^{\delta}$ are the standard RiemannLiouville derivatives. And it is assumed that the nonlinear source term $f$ satisfies $f \in C([0,1] \times[0,+\infty) \times[0,+\infty) ;[0$, $+\infty))$, and $\varphi_{p}$ is defined as $\varphi_{p}(s)=|s|^{p-2} s, p>1$.

Fractional differential equations have developed into a powerful tool to mathematically model and solve many real-world problems that arises in various fields such as phys- ics, chemistry, biology, and mechanics (see $[1,2]$ and references therein). Especially, since the turbulent flow was one of the fundamental problems in the field of fluid dynamics, Leibenson [3] and Esteban and Vazquez [4] proposed a mathematical model for one-dimensional, polytropic, and turbulent flow of a gas in a porous medium as

$$
u_{t}=\frac{\partial}{\partial x}\left(\frac{\partial u^{m}}{\partial x}\left|\frac{\partial u^{m}}{\partial x}\right|^{p-1}\right)
$$

where $u(t, x)$ was a scaled density at every point $x$ and time $t$. According to them, if $p=1$, the equation refers to the laminar filtration, in which case, it is often known as the fast diffusion equation if $0<m<1$ and the linear heat equation if $m=1$; otherwise, the porous media equation. On the other hand, the case $m=1, p \neq 1$ has been widely studied in nonNewtonian fluid dynamics (see [5] and the references therein). By some substitutions and tricks, this nonlinear problem was reduced into the following $p$-Laplacian equation to investigate the existence and properties of solutions to it $[6,7]$ :

$$
\left(\varphi_{p}\left(u^{\prime}(t)\right)\right)^{\prime}=f\left(t, u(t), u^{\prime}(t)\right) .
$$


Due to the significance of equation (3), many works have been studied to establish a lot of valuable existence and multiplicity results for various classes of higher-order and generalized $p$-Laplacian differential equations with integerorder derivatives [8-10]. For example, Guo et al. [8] dealt with the existence of at least three positive solutions to the quasilinear second-order differential equation

$$
\left(\varphi_{p}\left(u^{\prime}(t)\right)\right)^{\prime}+a(t) f(t, u(t))=0,0<t<1,
$$

subject to one of the following $m$-point boundary conditions:

$$
\varphi_{p}\left(u^{\prime}(0)\right)=\sum_{i=1}^{m-2} a_{i} \varphi_{p}\left(u^{\prime}\left(\xi_{i}\right)\right), u(1)=\sum_{i=1}^{m-2} b_{i} u\left(\xi_{i}\right)
$$

or

$$
u(0)=\sum_{i=1}^{m-2} a_{i} u\left(\xi_{i}\right), \varphi_{p}\left(u^{\prime}(1)\right)=\sum_{i=1}^{m-2} b_{i} \varphi_{p}\left(u^{\prime}\left(\xi_{i}\right)\right),
$$

by using the five functional fixed point theorem. Generalizing the results of the above studies for integer-order differential equations, many researchers have obtained great outcomes of the existence of solutions to single-term fractional differential equations with $p$-Laplacian operator (see [11-15]). From the representative results, Lv [11] established the existence and multiplicity of positive solutions to $m$-point boundary value problems of $p$-Laplacian fractional differential equations with a parameter:

$\left\{\begin{array}{l}D_{0+}^{\beta}\left(\varphi_{p}\left(D_{0+}^{\alpha} u(t)\right)\right)+\varphi_{p}(\lambda) f(t, u(t))=0,0<t<1,1<\alpha \leq 2,0<\beta \leq 1, \\ u(0)=0, D_{0+}^{\gamma} u(1)=\sum_{i=1}^{m-2} \xi_{i} D_{0+}^{\gamma} u\left(\eta_{i}\right), D_{0+}^{\alpha} u(0)=0,0<\gamma \leq 1,\end{array}\right.$

by using the theory of the fixed-point index in a cone and the monotone iterative technique. In addition, $\mathrm{Li}$ and $\mathrm{Qi}$ [12] improved the works of Guo et al. [8] to show the existence of positive solutions for the following multipoint boundary value problems of nonlinear fractional differential equations with $p$-Laplacian operator:

$$
\left\{\begin{array}{l}
{ }^{C} D_{0+}^{\beta}\left(\varphi_{p}\left({ }^{C} D_{0+}^{\alpha} u(t)\right)\right)+f(t, u(t))=0,0<t<1, n-1<\alpha \leq n, l-1<\beta \leq l, \\
\varphi_{p}\left({ }^{C} D_{0+}^{\alpha} u(0)\right)^{(i)}=0, i=1,2, \cdots, l-1, \\
\varphi_{p}\left({ }^{C} D_{0+}^{\alpha} u(1)\right)=\sum_{i=1}^{m-2} b_{i}\left[\varphi_{p}\left({ }^{C} D_{0+}^{\alpha} u\left(\xi_{i}\right)\right)\right], \\
u^{(i)}(0)=0, i=1,2, \cdots, n-1, \\
u(0)=\sum_{i=1}^{m-2} a_{i} u^{(j)}\left(\xi_{i}\right),
\end{array}\right.
$$

where $l>1, n \geq 2, j \in(0, \alpha)$ are integers and ${ }^{C} D_{0+}^{\alpha}{ }^{C} D_{0+}^{\beta}$ are the Caputo fractional derivatives. It is noted that the nota- tions $D_{0+}^{\alpha}$ and ${ }^{C} D_{0+}^{\alpha}$ appearing throughout the paper refer to the Riemann-Liouville and Caputo fractional derivatives, respectively, unless otherwise stated.

However, since the differential equation studied by Leibenson [3] is a multiterm one, it is important to extend the research results for single-term fractional differential equations to the case of multiterm. To do this, the existence of solutions to nonlinear multiterm fractional differential equations with a $p$-Laplacian operator have been studied by employing useful techniques in a nonlinear functional analysis such as the Banach contraction mapping principle, the Schauder fixed-point theorem, and the Krasnoselskii fixed-point theorem (see [16-20]). In particular, it is of great significance to study the case where $\alpha, \beta, \gamma$ are in $(0,1)$ or $(1,2)$, when considering the following fractional differential equation:

$$
D^{\beta}\left(\varphi_{p}\left(D^{\alpha} u\right)\right)(t)=f\left(t, u(t), D^{\gamma} u(t)\right)
$$

generalized equation (3) because of the inclusion of firstorder derivatives outside and inside of $\varphi_{p}$ in it. Here, $D^{\alpha}$, $D^{\beta}$, and $D^{\gamma}$ refer to fractional derivatives in several senses such as the Riemann-Liouville and the Caputo. Therefore, by using the coincidence degree theory, Chen et al. [16] studied two-point boundary value problems of fractional differential equations with $p$-Laplacian operator:

$$
\left\{\begin{array}{l}
{ }^{C} D_{0+}^{\beta}\left(\varphi_{p}\left({ }^{C} D_{0+}^{\alpha} x(t)\right)\right)=f\left(t, x(t),{ }^{C} D_{0+}^{\alpha} x(t)\right), t \in[0,1], 0<\alpha, \beta \leq 1, \\
{ }^{C} D_{0+}^{\alpha} x(0)={ }^{C} D_{0+}^{\alpha} x(1)=0,
\end{array}\right.
$$

to obtain new results on the existence of solutions to them. Besides, according to Liu et al. [17], the following fourpoint boundary value problems of $p$-Laplacian fractional differential equations with mixed fractional derivatives:

$$
\left\{\begin{array}{l}
D_{0+}^{\alpha}\left(\varphi_{p}\left({ }^{C} D_{0+}^{\beta} u(t)\right)\right)=f\left(t, u(t),{ }^{C} D_{0+}^{\beta} u(t)\right), t \in(0,1), 1<\alpha, \beta \leq 2, \\
{ }^{C} D_{0+}^{\beta} u(0)=u^{\prime}(0)=0, \\
u(1)=r_{1} u(\eta),{ }^{C} D_{0+}^{\beta} u(1)=r_{2}{ }^{C} D_{0+}^{\beta} u(\xi),
\end{array}\right.
$$

were considered to establish a lower and upper solution method which was used to prove the existence of positive solutions to them. After analyzing the results for nonlinear multiterm fractional differential equations with $p$-Laplacian operator, we have found that most of these previous works only dealt with some kinds of $p$-Laplacian equations that had certain rules for the fractional derivatives in nonlinear source terms and were limited to the cases of two-point, three-point, and four-point boundary conditions.

On the other hand, Dishlieva [21] and Li et al. [22] investigated the continuous dependency of solutions to their differential equations on initial conditions, barrier curves, and source terms in their studies but not on boundary conditions. 
This also implies that as far as we know, no works concerned with this issue for fractional differential equations with $p$ Laplacian operator can be done.

Motivated by the analysis mentioned above, this paper is aimed at studying some properties of positive solutions to $m$-point boundary value problems of nonlinear multiterm fractional differential equations with $p$-Laplacian operator including the existence, the uniqueness, and the continuous dependency on boundary conditions.

This study is organized as follows. In Section 2, we give some necessary definitions and preliminary results which will be used to prove our main results. In Section 3, we prove the existence and uniqueness of positive solutions to $p$-Laplacian fractional boundary value problem (1) and continuous dependence of them on the perturbations with respect to the coefficients in $m$-point boundary conditions. Finally, in Section 4, we illustrate our results by giving two examples.

Throughout the whole paper, it is also supposed that

$3<\alpha+\beta \leq 4, \gamma<\delta, \alpha-\delta-1>0, \sum_{i=1}^{m-2} \xi_{i} \eta_{i}^{\alpha-\delta-1}<1, \sum_{i=1}^{m-2} \zeta_{i} \eta_{i}^{\beta-1}<1$

\section{Preliminaries}

For the sake of convenience of the readers, some necessary definitions and lemmas will be presented here.

The Riemann-Liouville fractional integral and the Riemann-Liouville fractional derivative of order $\alpha>0$ of a function $f:(0, \infty) \rightarrow \mathbf{R}$ are defined as

$$
\begin{aligned}
\left(I_{0+}^{\alpha} f\right)(t) & :=\frac{1}{\Gamma(\alpha)} \int_{0}^{t}(t-s)^{\alpha-1} f(s) d s,\left(D_{0+}^{\alpha} f\right)(t) \\
& :=\frac{1}{\Gamma(n-\alpha)}\left(\frac{d}{d t}\right)^{n} \int_{0}^{t}(t-s)^{n-\alpha-1} f(s) d s,
\end{aligned}
$$

where $n=[\alpha]+1$, provided that the right-hand sides are pointwise defined on $(0, \infty)$ (see [23]).

Lemma 1 (see [24]). Assume that $u \in C(0,1) \cap L(0,1)$ with a fractional derivative of order $\alpha>0$ that belongs to $C(0,1) \cap$ $L(0,1)$. Then,

$$
I_{0+}^{\alpha} D_{0+}^{\alpha} u(t)=u(t)+C_{1} t^{\alpha-1}+C_{2} t^{\alpha-2}+\cdots+C_{N} t^{\alpha-N},
$$

for some $C_{i} \in \mathbf{R}, i=1,2, \cdots, N$, where $N$ is the smallest integer greater than or equal to $\alpha$.

Since $1<\alpha \leq 2,0<\gamma<\delta<1, \alpha-\delta>1$, it is obvious that $1<\alpha-\gamma<2$. And putting $A:=1-\sum_{i=1}^{m-2} \xi_{i} \eta_{i}^{\alpha-\gamma-1}$ and $B:=1-$ $\sum_{i=1}^{m-2} \zeta_{i} \eta_{i}^{\beta-1}$, the following lemmas hold.
Lemma 2 (see [11]). Let $y \in C[0,1]$. Then, the fractional differential equation

$$
\left\{\begin{array}{l}
D_{0+}^{\alpha-\gamma} u(t)+y(t)=0,0<t<1, \\
u(0)=0, D_{0+}^{\delta-\gamma} u(1)=\sum_{i=1}^{m-2} \xi_{i} D_{0+}^{\delta-\gamma} u\left(\eta_{i}\right),
\end{array}\right.
$$

has a unique solution which is given by

$$
u(t)=\int_{0}^{1} G(t, s) y(s) d s
$$

where $G(t, s)=G_{1}(t, s)+G_{2}(t, s)$,

in which

$$
\begin{aligned}
G_{1}(t, s)= & \frac{1}{\Gamma(\alpha-\gamma)}\left\{\begin{array}{l}
t^{\alpha-\gamma-1}(1-s)^{\alpha-\delta-1}-(t-s)^{\alpha-\gamma-1}, 0 \leq s \leq t \leq 1, \\
t^{\alpha-\gamma-1}(1-s)^{\alpha-\delta-1}, 0 \leq t \leq s \leq 1,
\end{array}\right. \\
G_{2}(t, s)= & \frac{t^{\alpha-\gamma-1}}{A \Gamma(\alpha-\gamma)}\left[\sum_{s<\eta_{i}} \xi_{i}\left[\eta_{i}^{\alpha-\delta-1}(1-s)^{\alpha-\delta-1}-\left(\eta_{i}-s\right)^{\alpha-\delta-1}\right]\right. \\
& \left.+\sum_{s \geq \eta_{i}} \xi_{i} \eta_{i}^{\alpha-\delta-1}(1-s)^{\alpha-\delta-1}\right], t, s \in[0,1] .
\end{aligned}
$$

Lemma 3 (see [11]). If $\sum_{i=1}^{m-2} \xi_{i} \eta_{i}^{\alpha-\delta-1}$, then the function $G(t, s)$ in Lemma 2 satisfies the following conditions:

(i) $G(t, s)>0$, for any $t, s \in(0,1)$

(ii) $G(t, s) \leq G_{*}(s, s)$, for any $t, s \in[0,1]$

where

$$
G_{*}(s, s)=\frac{1}{A \Gamma(\alpha-\gamma)}(1-s)^{\alpha-\delta-1} .
$$

Lemma 4 (see [25]). Let $z \in C[0,1]$. Then, the fractional differential equation

$$
\left\{\begin{array}{l}
D_{0+}^{\beta} v(t)+z(t)=0 \\
v(0)=0, v(1)=\sum_{i=1}^{m-2} \zeta_{i} v\left(\eta_{i}\right)
\end{array}\right.
$$

has a unique solution which is given by

$$
v(t)=\int_{0}^{1} H(t, s) z(s) d s
$$


where $H(t, s)=H_{1}(t, s)+H_{2}(t, s)$, in which

$$
\begin{aligned}
H_{1}(t, s)= & \frac{1}{\Gamma(\beta)}\left\{\begin{array}{l}
t^{\beta-1}(1-s)^{\beta-1}-(t-s)^{\beta-1}, 0 \leq s \leq t \leq 1, \\
t^{\beta-1}(1-s)^{\beta-1}, 0 \leq t \leq s \leq 1,
\end{array}\right. \\
H_{2}(t, s)= & \frac{t^{\beta-1}}{B \Gamma(\beta)}\left[\sum_{s<\eta_{i}} \zeta_{i}\left[\eta_{i}^{\beta-1}(1-s)^{\beta-1}-\left(\eta_{i}-s\right)^{\beta-1}\right]\right. \\
& \left.+\sum_{s \geq \eta_{i}} \zeta_{i} \eta_{i}^{\beta-1}(1-s)^{\beta-1}\right], t, s \in[0,1] .
\end{aligned}
$$

Lemma 5 (see [25]). If $\sum_{i=1}^{m-2} \zeta_{i} \eta_{i}^{\beta-1}<1$, then the function $H(t, s)$ in Lemma 4 satisfies the following conditions:

(i) $H(t, s)>0$, for any $t, s \in(0,1)$

(ii) $H(t, s) \leq H_{*}(s, s)$, for any $t, s \in[0,1]$

where

$$
H_{*}(s, s)=\frac{1}{B \Gamma(\beta)}(1-s)^{\beta-1}
$$

The following properties of $\varphi_{p}(\cdot)$ which will be used later can be found in [26]:

(i) If $1<p<2, x y>0$, and $|x|,|y| \geq m>0$, then

$$
\left|\varphi_{p}(x)-\varphi_{p}(y)\right| \leq(p-1) m^{p-2}|x-y|
$$

(ii) If $p>2,|x|,|y| \leq M$, then

$$
\left|\varphi_{p}(x)-\varphi_{p}(y)\right| \leq(p-1) M^{p-2}|x-y|
$$

\section{Main Results}

3.1. Existence and Uniqueness of Positive Solutions to Problem (1)

Definition 6. A function $x \in\left\{u \mid u \in C[0,1], D_{0+}^{\alpha} u \in C[0,1]\right.$, $\left.D_{0+}^{\beta}\left(\varphi_{p}\left(D_{0+}^{\alpha} u\right)\right) \in C[0,1]\right\}$ is called a solution of problem (1) if it satisfies the fractional differential equation and the boundary conditions of (1).

Lemma 7. If the function $x$ is a solution of problem (1), then $u(t):=D_{0+}^{\gamma} x(t)$ is a solution of the integral equation

$$
u(t)=\int_{0}^{1} G(t, s) \varphi_{q}\left(\int_{0}^{1} H(s, \tau) f\left(\tau, I_{0+}^{\gamma} u(\tau), u(\tau)\right) d \tau\right) d s
$$

in $C[0,1]$ and conversely, if $u \in C[0,1]$ is a solution of the integral equation (25), then $x(t)=I_{0+}^{\gamma} u(t)$ is a solution of problem (1), where $q$ is a number such that $(1 / p)+(1 / q)=1$.
Proof. Let $x$ be a solution of the problem (1). Then, since $x \in C[0,1], D_{0+}^{\alpha} x \in C[0,1]$, by using Lemma 1 , it can be easily seen that

$$
x(t)=I_{0+}^{\alpha} D_{0+}^{\alpha} x(t)+c_{1} t^{\alpha-1}+c_{2} t^{\alpha-2} .
$$

The upper continuities of the functions $x(t), I_{0+}^{\alpha} D_{0+}^{\alpha} x(t)$ at $t=0$ provide us that $c_{2}=0$. Applying $D_{0+}^{\gamma}$ on both sides, we have

$$
\begin{aligned}
D_{0+}^{\gamma} x(t) & =D_{0+}^{\gamma} I_{0+}^{\alpha} D_{0+}^{\alpha} x(t)+D_{0+}^{\gamma} c_{1} t^{\alpha-1} \\
& =I_{0+}^{\alpha-\gamma} D_{0+}^{\alpha} x(t)+\frac{\Gamma(\alpha)}{\Gamma(\alpha-\gamma)} c_{1} t^{\alpha-\gamma-1} .
\end{aligned}
$$

Combining with $D_{0+}^{\alpha} x \in C[0,1], \alpha-\gamma-1>0$, we can see that $D_{0+}^{\gamma} x \in C[0,1]$. Since $x \in C[0,1], D_{0+}^{\gamma} x \in C[0,1]$, Lemma 1 yields that

$$
x(t)=I_{0+}^{\gamma} D_{0+}^{\gamma} x(t)+c_{3} t^{\gamma-1} .
$$

Therefore, it follows from the upper continuities of the functions $x(t), I_{0+}^{\gamma} D_{0+}^{\gamma} x(t)$ at $t=0$ that $c_{3}=0$. This implies that by putting $u(t):=D_{0+}^{\gamma} x(t)$, it holds that $x(t)=I_{0+}^{\gamma} u(t)$. Thus, we can know that $u$ is a solution of the following problem:

$$
\left\{\begin{array}{l}
D_{0+}^{\beta}\left(\varphi_{p}\left(D_{0+}^{\alpha-\gamma} u(t)\right)\right)=f\left(t, I_{0+}^{\gamma} u(t), u(t)\right), 0<t<1, \\
u(0)=0, D_{0+}^{\delta-\gamma} u(1)=\sum_{i=1}^{m-2} \xi_{i} D_{0+}^{\delta-\gamma} u\left(\eta_{i}\right), \\
D_{0+}^{\alpha-\gamma} u(0)=0, \varphi_{p}\left(D_{0+}^{\alpha-\gamma} u(1)\right)=\sum_{i=1}^{m-2} \zeta_{i} \varphi_{p}\left(D_{0+}^{\alpha-\gamma} u\left(\eta_{i}\right)\right) .
\end{array}\right.
$$

Put $w(t):=D_{0+}^{\alpha-\gamma} u(t)$. Lemma 2 shows that

$$
u(t)=-\int_{0}^{1} G(t, s) w(s) d s
$$

Also, by applying Lemma 4 , the notation $v(t)=\varphi_{p}(w(t))$ gives us that

$$
v(t)=-\int_{0}^{1} H(t, s) f\left(s, I_{0+}^{\gamma} u(s), u(s)\right) d s
$$

Since $\varphi_{p}^{-1}=\varphi_{q}$, by equations (30) and (31), we obtain

$$
u(t)=\int_{0}^{1} G(t, s) \varphi_{q}\left(\int_{0}^{1} H(s, \tau) f\left(\tau, I_{0+}^{\gamma} u(\tau), u(\tau)\right) d \tau\right) d s .
$$


Conversely, let the function $u \in C[0,1]$ be a solution of integral equation (25). From this, by putting $x(t):=I_{0+}^{\gamma} u(t)$, it can be also obtained that $x \in C[0,1]$. Denote as follows:

$$
z(t):=f\left(t, I_{0+}^{\gamma} u(t), u(t)\right), v(t):=\varphi_{q}\left(\int_{0}^{1} H(t, s) z(s) d s\right) .
$$

From the continuities of the functions $u, f$, and $H$, it is obvious that $z \in C[0,1], v \in C[0,1]$. The definition of the function $G(t, s)$ implies that

$$
\begin{aligned}
u(t)= & \int_{0}^{1} G(t, \quad s) v(s) d s \\
= & -I_{0+}^{\alpha-\gamma} v(t)-\frac{\Gamma(\alpha-\delta)}{A \Gamma(\alpha-\gamma)} \\
& \cdot\left[\left.\sum_{i=1}^{m-2} \xi_{i} I_{0+}^{\alpha-\delta} v(t)\right|_{t=\eta_{i}}-\left.I_{0+}^{\alpha-\delta} v(t)\right|_{t=1}\right] t^{\alpha-\gamma-1},
\end{aligned}
$$

so, we have

$$
D_{0+}^{\alpha} x(t)=D_{0+}^{\alpha-\gamma} u(t)=v(t) .
$$

This means that $D_{0+}^{\alpha} x \in C[0,1]$. Also, combining the definition of the function $H(t, s)$ with the relation

$$
\begin{aligned}
D_{0+}^{\beta}\left(\varphi_{p}\left(D_{0+}^{\alpha} x(t)\right)\right) & =D_{0+}^{\beta}\left(\varphi_{p}(v(t))\right) \\
& =D_{0+}^{\beta}\left(\int_{0}^{1} H(t, s) z(s) d s\right),
\end{aligned}
$$

we can get

$$
\begin{aligned}
& \int_{0}^{1} H(t, \quad s) z(s) d s \\
& \quad=-I_{0+}^{\beta} z(t)-\frac{1}{B}\left[\left.\sum_{i=1}^{m-2} \zeta_{i} I_{0+}^{\beta} z(t)\right|_{t=\eta_{i}}-\left.I_{0+}^{\beta} z(t)\right|_{t=1}\right] t^{\beta-1} .
\end{aligned}
$$

This yields that

$$
D_{0+}^{\beta}\left(\varphi_{p}\left(D_{0+}^{\alpha} x(t)\right)\right)=z(t),
$$

and therefore, it holds that $D_{0+}^{\beta}\left(\varphi_{p}\left(D_{0+}^{\alpha} x\right)\right) \in C[0,1]$. Since equations (15) and (19) have unique solutions by Lemmas 2 and $4, u$, the solution of integral equation (25), satisfies the fractional differential equation and the boundary conditions of the problem (29). To sum up, it can be proved that $x$ is a solution of problem (1).

Let $E$ be the Banach space $C[0,1]$ equipped with the norm $\|u\|=\max _{0 \leq t \leq 1}|u(t)|$ and put $P:=\{u \in E \mid u(t) \geq 0, t \in[0,1]\}$. Since $\forall u \in P, \forall t \in[0,1], I_{0+}^{\gamma} u(t)=(1 / \Gamma(\gamma)) \int_{0}^{t}(t-s)^{\gamma-1} u(s) d s$ $\geq 0$, it holds that $I_{0+}^{\gamma} u \in P$. Therefore, define an operator $T$ as

$$
T u(t):=\int_{0}^{1} G(t, s) \varphi_{q}\left(\int_{0}^{1} H(s, \tau) f\left(\tau, I_{0+}^{\gamma} u(\tau), u(\tau)\right) d \tau\right) d s, u \in P,
$$

then, it satisfies $T(P) \subset P$. This implies that integral equation (25) has a solution in $P$ if and only if operator $T$ has a fixed point. It is also obvious that the fixed point of operator $T$ is a solution of integral equation (25) in $P$.

Put $\bar{M}:=[(\alpha-\delta) A \Gamma(\alpha-\gamma)]^{p-1} B \Gamma(\beta)$. The following hypotheses will be used throughout the paper.

(H1) There exist $a, b, c \in L[0,1]$ such that

$$
\begin{gathered}
M_{a}:=\int_{0}^{1} a(t) d t>0, M_{b}:=\int_{0}^{1} b(t) d t>0, M_{c}:=\int_{0}^{1} c(t) d t>0, \\
\frac{M_{b}}{\Gamma(\gamma+1)^{p-1}}+M_{c}<\bar{M}, \\
\forall(t, x, y) \in[0,1] \times\left[0, R_{1}\right] \times\left[0, R_{2}\right], \\
f(t, x, y)<a(t)+b(t) x^{p-1}+c(t) y^{p-1},
\end{gathered}
$$

where $R_{2}=\left(M_{a} /\left(\bar{M}-\left(M_{b} /\left(\Gamma(\gamma+1)^{p-1}\right)\right)-M_{c}\right)\right)^{q-1} \quad$ and $R_{1}=R_{2} /(\Gamma(\gamma+1))$

(H2) There exist $L_{1}, L_{2}>0$ such that for any $t \in[0,1]$ and any $\left(x_{1}, y_{1}\right),\left(x_{2}, y_{2}\right) \in\left[0, R_{1}\right] \times\left[0, R_{2}\right]$,

$$
\left|f\left(t, x_{1}, y_{1}\right)-f\left(t, x_{2}, y_{2}\right)\right| \leq L_{1}\left|x_{1}-x_{2}\right|+L_{2}\left|y_{1}-y_{2}\right|
$$

(H3) There exist $\bar{m}, \mu>0$ such that for any $(t, x, y) \epsilon$ $[0,1] \times\left[0, R_{1}\right] \times\left[0, R_{2}\right]$,

$$
f(t, x, y) \geq \bar{m} t^{\mu-1}
$$

Lemma 8. Assume that the hypothesis (H1) is satisfied. Then, it holds that

$$
T\left(B_{R_{2}}\right) \subset B_{R_{2}}
$$

where $B_{R_{2}}=\left\{u \in P \mid\|u\| \leq R_{2}\right\}$.

Proof. Since $T\left(B_{R_{2}}\right) \subset P$, it is sufficient to prove that $\forall u \in B_{R_{2}}$, $\|T u\| \leq R_{2}$. It can be easily evaluated that for any $t \in[0,1]$,

$$
\begin{aligned}
I_{0+}^{\gamma} u(t) & =\frac{1}{\Gamma(\gamma)} \int_{0}^{t}(t-s)^{\gamma-1} u(s) d s \leq \frac{R_{2}}{\Gamma(\gamma)} \int_{0}^{t}(t-s)^{\gamma-1} d s \\
& =\frac{R_{2}}{\Gamma(\gamma+1)} \cdot t^{\gamma} \leq R_{1} .
\end{aligned}
$$


By using Lemma 5 and hypothesis ( $\mathrm{H} 1)$, we have

$$
\begin{aligned}
|T u(t)|= & \int_{0}^{1} G(t, s) \varphi_{q}\left(\int_{0}^{1} H(s, \tau) f\left(\tau, I_{0+}^{\gamma} u(\tau), u(\tau)\right) d \tau\right) d s \\
\leq & \int_{0}^{1} G(t, s) \varphi_{q}\left(\operatorname { m a x } _ { s \in [ 0 , 1 ] } H _ { * } ( s , s ) \int _ { 0 } ^ { 1 } \left(a(\tau)+b(\tau)\left(I_{0+}^{\gamma} u(\tau)\right)^{p-1}\right.\right. \\
& \left.\left.+c(\tau) u(\tau)^{p-1}\right) d \tau\right) d s \\
\leq & \int_{0}^{1} G(t s) \varphi_{q}\left(\frac{1}{B \Gamma(\beta)}\left(M_{a}+\left(\frac{M_{b}}{\Gamma(\gamma+1)^{p-1}}+M_{c}\right) R_{2}^{p-1}\right)\right) d s .
\end{aligned}
$$

Applying the properties of the function $G(t, s)$ indicated in Lemma 3, the following holds:

$$
\begin{aligned}
|T u(t)| & \leq\left(\frac{1}{B \Gamma(\beta)}\left(M_{a}+\left(\frac{M_{b}}{\Gamma(\gamma+1)^{p-1}}+M_{c}\right) R_{2}^{p-1}\right)\right)^{q-1} \int_{0}^{1} G_{*}(s, s) d s \\
& =\frac{\left(M_{a}+\left(\left(M_{b} /\left(\Gamma(\gamma+1)^{p-1}\right)\right)+M_{c}\right) R_{2}^{p-1}\right)^{q-1}}{B^{q-1} \Gamma(\beta)^{q-1} A(\alpha-\delta) \Gamma(\alpha-\gamma)} .
\end{aligned}
$$

The relation $(1 / p)+(1 / q)=1$ shows that $p-1$ is an inverse power of $q-1$. So, it can be rewritten as

$$
|T u(t)| \leq\left(\left(M_{a}+\left(\frac{M_{b}}{\Gamma(\gamma+1)^{p-1}}+M_{c}\right) R_{2}^{p-1}\right) / \bar{M}\right)^{q-1} .
$$

Since it is satisfied for $R_{2}$ that

$$
M_{a}=\left(\bar{M}-\frac{M_{b}}{\Gamma(\gamma+1)^{p-1}}-M_{c}\right) R_{2}^{p-1}
$$

we can get

$$
|T u(t)| \leq R_{2}
$$

This concludes the lemma.

Lemma 9. If the hypothesis (H3) holds, then for any $t \in[0,1]$ and any $u \in B_{R_{2}}$,

$$
\int_{0}^{1} H(t, s) f\left(s, I_{0+}^{\gamma} u(s), u(s)\right) d s \geq K_{0} t^{\beta-1}
$$

where $K_{0}=((\bar{m} \Gamma(\mu)) /(B \Gamma(\beta+\mu))) \sum_{i=1}^{m-2} \zeta_{i}\left(\eta_{i}^{\beta-1}-\eta_{i}^{\beta-1+\mu}\right)$.

Proof. It follows from Lemma 4 and hypothesis (H3) that

$$
\begin{aligned}
& \int_{0}^{1} H(t, s) f\left(s, I_{0+}^{\gamma} u(s), u(s)\right) d s \\
& \quad \geq \bar{m}\left(\int_{0}^{1} H_{1}(t, s) s^{\mu-1} d s+\int_{0}^{1} H_{2}(t, s) s^{\mu-1} d s\right) .
\end{aligned}
$$

By simple calculation, the integration terms in the right side of inequality (51) can be estimated as

$$
\begin{aligned}
\int_{0}^{1} H_{1}(t, s) s^{\mu-1} d s= & \frac{1}{\Gamma(\beta)}\left[t^{\beta-1} \int_{0}^{1}(1-s)^{\beta-1} s^{\mu-1} d s\right. \\
& \left.-\int_{0}^{t}(t-s)^{\beta-1} s^{\mu-1} d s\right] \\
= & \frac{1}{\Gamma(\beta)}\left[t^{\beta-1} B(\mu, \beta)-t^{\beta-1+\mu} B(\mu, \beta)\right] \\
= & \frac{\Gamma(\mu)}{\Gamma(\beta+\mu)}\left[t^{\beta-1}-t^{\beta-1+\mu}\right] \geq 0, \\
\int_{0}^{1} H_{2}(t, s) s^{\mu-1} d s= & \frac{t^{\beta-1}}{B \Gamma(\beta)} \sum_{i=1}^{m-2} \zeta_{i}\left[\eta_{i}^{\beta-1} \int_{0}^{1}(1-s)^{\beta-1} s^{\mu-1} d s\right. \\
& \left.-\int_{0}^{\eta_{i}}\left(\eta_{i}-s\right)^{\beta-1} s^{\mu-1} d s\right] \\
= & \frac{t^{\beta-1}}{B \Gamma(\beta)} \sum_{i=1}^{m-2} \zeta_{i}\left[\eta_{i}^{\beta-1} B(\mu, \beta)-\eta_{i}^{\beta-1+\mu} B(\mu, \beta)\right] \\
= & {\left[\frac{\Gamma(\mu)}{B \Gamma(\beta+\mu)} \sum_{i=1}^{m-2} \zeta_{i}\left(\eta_{i}^{\beta-1}-\eta_{i}^{\beta-1+\mu}\right)\right] t^{\beta-1} . }
\end{aligned}
$$

These estimations provide us the conclusion (50).

Denote $K_{1}:=(1 / \Gamma(\beta+1))\left[1+(1 / B) \sum_{i=1}^{m-2} \zeta_{i}\left(\eta_{i}^{\beta-1}-\eta_{i}^{\beta}\right)\right]$. Then, it can be found in [25] that for any $t \in[0,1]$,

$$
\int_{0}^{1} H(t, s) d s \leq K_{1} t^{\beta-1}
$$

Theorem 10. Suppose that $p>2$ and hypotheses (H1)-(H3) are satisfied. If

$$
\frac{(q-1) K_{1}}{A(\alpha-\delta) \Gamma(\alpha-\gamma) K_{0}^{2-q}}\left(\frac{L_{1}}{\Gamma(\gamma+1)}+L_{2}\right)<1,
$$

then, integral equation (25) has a unique solution in $B_{R_{2}}$.

Proof. To prove this theorem, operators $T_{0}, T_{1}$ are defined as

$$
\begin{aligned}
T_{0} u(t) & :=\varphi_{q}\left(\int_{0}^{1} H(t, s) f\left(s, I_{0+}^{\gamma} u(s), u(s)\right) d s\right), T_{1} u(t) \\
& :=\int_{0}^{1} G(t, s) u(s) d s .
\end{aligned}
$$

Obviously, we can see that for any $u \in B_{R_{2}}, T u(t)=$ $\left(T_{1} \mathrm{oT}_{0} u\right)(t)$, and $T u(0)=0$. Because $p>2$, it is satisfied that 
$1<q<2$. So, by employing Lemma 8 , Lemma 9 , and property (23), we can obtain that for any $u, v \in B_{R_{2}}$ and any $t \in(0,1]$,

$$
\begin{aligned}
\mid T_{0} u(t) & -T_{0} v(t) \mid \\
= & \mid \varphi_{q}\left(\int_{0}^{1} H(t, s) f\left(s, I_{0+}^{\gamma} u(s), u(s)\right) d s\right) \\
& -\varphi_{q}\left(\int_{0}^{1} H(t, s) f\left(s, I_{0+}^{\gamma} v(s), v(s)\right) d s\right) \mid \\
\leq & (q-1)\left(K_{0} t^{\beta-1}\right)^{q-2} \mid \int_{0}^{1} H(t, s) f\left(s, I_{0+}^{\gamma} u(s), u(s)\right) d s \\
& -\int_{0}^{1} H(t, s) f\left(s, I_{0+}^{\gamma} v(s), v(s)\right) d s \mid \\
\leq & (q-1)\left(K_{0} t^{\beta-1}\right)^{q-2} \int_{0}^{1} H(t, s)\left(L_{1}\left|I_{0+}^{\gamma} u(s)-I_{0+}^{\gamma} v(s)\right|\right. \\
& \left.+L_{2}|u(s)-v(s)|\right) d s \\
\leq & (q-1)\left(K_{0} t^{\beta-1}\right)^{q-2} \int_{0}^{1} H(t, s)\left(\frac{L_{1}}{\Gamma(\gamma+1)}\|u-v\|\left\|+L_{2}\right\| u-v \|\right) d s \\
\leq & (q-1)\left(K_{0} t^{\beta-1}\right)^{q-2}\left(\frac{L_{1}}{\Gamma(\gamma+1)}+L_{2}\right)\|u-v\| \int_{0}^{1} H(t, s) d s .
\end{aligned}
$$

Substituting inequality (53) into (56), we have

$$
\begin{aligned}
\left|T_{0} u(t)-T_{0} v(t)\right| \leq & (q-1) K_{0}^{q-2} K_{1} \\
& \cdot\left(\frac{L_{1}}{\Gamma(\gamma+1)}+L_{2}\right)\|u-v\| t^{(\beta-1)(q-1)} .
\end{aligned}
$$

Thus, it holds that

$$
\begin{aligned}
|T u(t)-T v(t)|= & \left|\int_{0}^{1} G(t, s)\left(T_{0} u\right)(s) d s-\int_{0}^{1} G(t, s)\left(T_{0} v\right)(s) d s\right| \\
\leq & (q-1) K_{0}^{q-2} K_{1}\left(\frac{L_{1}}{\Gamma(\gamma+1)}+L_{2}\right)\|u-v\| \\
& \cdot \int_{0}^{1} G(t, s) s^{(\beta-1)(q-1)} d s .
\end{aligned}
$$

Since $(\beta-1)(q-1)>0$, by Lemma 3 , we can get

$$
\begin{aligned}
\int_{0}^{1} G(t, s) s^{(\beta-1)(q-1)} d s & \leq \int_{0}^{1} G(t, s) d s \leq \int_{0}^{1} G_{*}(s, s) d s \\
& =\frac{1}{A(\alpha-\delta) \Gamma(\alpha-\gamma)} .
\end{aligned}
$$

This yields that

$\|T u-T v\| \leq \frac{(q-1) K_{1}}{A(\alpha-\delta) \Gamma(\alpha-\gamma) K_{0}^{2-q}}\left(\frac{L_{1}}{\Gamma(\gamma+1)}+L_{2}\right)\|u-v\|$.

Combining inequalities (54) and (60) with Lemma 8, the operator $T: B_{R_{2}} \rightarrow B_{R_{2}}$ is a contraction mapping and the Banach contraction mapping principle provides us that it has a unique fixed point in $B_{R_{2}}$. So, integral equation (25) has a unique solution in $B_{R_{2}}$.

Lemma 11. Assume that the hypothesis (H1) holds. Then, for any $u \in B_{R_{2}}$ and any $t \in[0,1]$, it is satisfied that

$$
\int_{0}^{1} H(t, s) f\left(s, I_{0+}^{\gamma} u(s), u(s)\right) d s \leq M_{0},
$$

where

$$
M_{0}=\frac{M_{a}+\left(\left(M_{b} /\left(\Gamma(\gamma+1)^{p-1}\right)\right)+M_{c}\right) R_{2}^{p-1}}{B \Gamma(\beta)} .
$$

Proof. In a similar way to the proof of Lemma 8, it can be easily proved that

$$
\begin{aligned}
& \int_{0}^{1} H(t, s) f\left(s, I_{0+}^{\gamma} u(s), u(s)\right) d s \\
& \quad \leq \max _{s \in[0,1]} H_{*}(s, s) \int_{0}^{1} f\left(s, I_{0+}^{\gamma} u(s), u(s)\right) d s \\
& \quad \leq \frac{1}{B \Gamma(\beta)} \int_{0}^{1}\left(a(s)+b(s)\left(I_{0+}^{\gamma} u(s)\right)^{p-1}+c(s) u(s)^{p-1}\right) d s \\
& \quad \leq \frac{1}{B \Gamma(\beta)}\left(\int_{0}^{1} a(s) d s+R_{1}^{p-1} \int_{0}^{1} b(s) d s+R_{2}^{p-1} \int_{0}^{1} c(s) d s\right)=M_{0} .
\end{aligned}
$$

Theorem 12. Suppose that $1<p<2$ and hypotheses (H1) and (H2) hold. If

$$
\frac{(q-1) R_{2}^{2-p}}{\beta \bar{M}}\left(\frac{L_{1}}{\Gamma(\gamma+1)}+L_{2}\right)<1,
$$

then, integral equation (25) has a unique solution in $B_{R_{2}}$.

Proof. In the case $1<p<2$, due to $(1 / p)+(1 / q)=1$, it holds that $q>2$. Similar to Theorem 10, defining operators $T_{0}$, $T_{1}$ with Lemma 8, Lemma 11, and property (24) give us that for any $u, v \in B_{R_{2}}$ and any $t \in(0,1]$,

$$
\begin{aligned}
&\left|T_{0} u(t)-T_{0} v(t)\right| \\
&=\mid \varphi_{q}\left(\int_{0}^{1} H(t, s) f\left(s, I_{0+}^{\gamma} u(s), u(s)\right) d s\right) \\
& \quad-\varphi_{q}\left(\int_{0}^{1} H(t, s) f\left(s, I_{0+}^{\gamma} v(s), v(s)\right) d s\right) \mid \\
& \leq(q-1) M_{0}^{q-2} \int_{0}^{1} H(t, s)\left|f\left(s, I_{0+}^{\gamma} u(s), u(s)\right)-f\left(s, I_{0+}^{\gamma} v(s), v(s)\right)\right| d s \\
& \leq(q-1) M_{0}^{q-2} \int_{0}^{1} H(t, s)\left(L_{1}\left|I_{0+}^{\gamma} u(s)-I_{0+}^{\gamma} v(s)\right|+L_{2}|u(s)-v(s)|\right) d s \\
& \leq(q-1) M_{0}^{q-2} \int_{0}^{1} H(t, s)\left(\frac{L_{1}}{\Gamma(\gamma+1)}\|u-v\|+L_{2}\|u-v\|\right) d s \\
& \leq(q-1) M_{0}^{q-2}\left(\frac{L_{1}}{\Gamma(\gamma+1)}+L_{2}\right)\|u-v\| \int_{0}^{1} H_{*}(s, s) d s \\
&= \frac{(q-1) M_{0}^{q-2}}{B \Gamma(\beta+1)}\left(\frac{L_{1}}{\Gamma(\gamma+1)}+L_{2}\right)\|u-v\| .
\end{aligned}
$$


So, we have

$$
\begin{aligned}
|T u(t)-T v(t)|= & \left|\int_{0}^{1} G(t, s)\left(T_{0} u\right)(s) d s-\int_{0}^{1} G(t, s)\left(T_{0} v\right)(s) d s\right| \\
\leq & \int_{0}^{1} G(t, s)\left|T_{0} u(s)-T_{0} v(s)\right| d s \\
\leq & \frac{(q-1) M_{0}^{q-2}}{B \Gamma(\beta+1)}\left(\frac{L_{1}}{\Gamma(\gamma+1)}+L_{2}\right)\|u-v\| \\
& \cdot \int_{0}^{1} G(t, s) d s \\
\leq & \frac{(q-1) M_{0}^{q-2}}{(\alpha-\delta) A B \Gamma(\alpha-\gamma) \Gamma(\beta+1)} \\
& \cdot\left(\frac{L_{1}}{\Gamma(\gamma+1)}+L_{2}\right)\|u-v\| .
\end{aligned}
$$

Obviously, for $M_{0}$, the following holds:

$\frac{M_{0}^{q-2}}{(\alpha-\delta) A B \Gamma(\alpha-\gamma) \Gamma(\beta+1)}=\frac{1}{\beta \bar{M}^{q-1}} \cdot\left(\bar{M} R_{2}^{p-1}\right)^{q-2}=\frac{R_{2}^{2-p}}{\beta \bar{M}}$.

Therefore, by employing inequality (64), equality (67), and Lemma 8, the operator $T: B_{R_{2}} \rightarrow B_{R_{2}}$ is a contraction mapping. It follows from the Banach contraction mapping principle that the operator $T$ has a unique fixed point in $B_{R_{2}}$. This completes the proof.

Remark 13. Theorems 10 and 12 show the existence and uniqueness of solutions to integral equation (25) in the cases $p>2$ and $1<p<2$, respectively. The Banach contraction mapping principle, employed in the proofs of these theorems, guarantees that the iterative sequences can be constructed to converge to the exact solution of integral equation (25). In other words, the iterative sequence $\left\{u_{n}\right\}_{n=0}^{\infty}$ initialized at any point $u_{0} \in B_{R_{2}}$ and defined by

$$
u_{n+1}=T u_{n}, n=0,1,2, \cdots
$$

converges to the exact solution of integral equation (25), $u$, in $B_{R_{2}}$. This provides the possibility to obtain the approximate solution $u_{n}$ of integral equation (25) by using Theorems 10 and 12 .

Theorem 14. Problem (1) has a unique solution in $\Omega$ if and only if the solution to integral equation (25) uniquely exists in $B_{R_{2}}$, where $\Omega=I_{0+}^{\gamma}\left(B_{R_{2}}\right)$. In particular, if hypothesis (H3) is satisfied, then the solution to problem (1) is positive.
Proof. By Lemma 7, for $x$, a solution to problem (1), and $u$ satisfying integral equation (25), the following relation holds:

$$
x(t)=I_{0+}^{\gamma} u(t), t \in[0,1] .
$$

This proves the first part of the theorem.

For the second part, it is necessary that if hypothesis (H3) holds, then

$$
\forall t \in(0,1], x(t)>0
$$

To prove this, we must see if the following holds:

$$
\forall t \in(0,1], u(t)>0
$$

Since $u \in B_{R_{2}}$, using Lemma 9 , it can be easily obtained that

$$
\begin{aligned}
u(t) & \geq K_{0}^{q-1} \int_{0}^{1} G(t, s) s^{(\beta-1)(q-1)} d s \\
& =K_{0}^{q-1}\left[\int_{0}^{1} G_{1}(t, s) s^{(\beta-1)(q-1)} d s+\int_{0}^{1} G_{2}(t, s) s^{(\beta-1)(q-1)} d s\right] .
\end{aligned}
$$

Considering that $G_{1}(t, s) \geq 0$ and $(\beta-1)(q-1)>0$, we can get

$$
\forall t \in[0,1], \int_{0}^{1} G_{1}(t, s) s^{(\beta-1)(q-1)} d s \geq 0 .
$$

From the definition of function $G_{2}(t, s)$, we have

$$
\begin{aligned}
\int_{0}^{1} G_{2}(t, s) s^{(\beta-1)(q-1)} d s & \\
= & \frac{t^{\alpha-\gamma-1}}{A \Gamma(\alpha-\gamma)} \sum_{i=1}^{m-2} \xi_{i}\left[\eta_{i}^{\alpha-\delta-1} \int_{0}^{1}(1-s)^{\alpha-\delta-1} s^{(\beta-1)(q-1)} d s\right. \\
& \left.-\int_{0}^{\eta_{i}}\left(\eta_{i}-s\right)^{\alpha-\delta-1} s^{(\beta-1)(q-1)} d s\right] \\
= & {\left[\frac{B(\alpha-\delta,(\beta-1)(q-1)+1)}{A \Gamma(\alpha-\gamma)}\right.} \\
& \left.\cdot \sum_{i=1}^{m-2} \xi_{i}\left(\eta_{i}^{\alpha-\delta-1}-\eta_{i}^{\alpha-\delta+(\beta-1)(q-1)}\right)\right] t^{\alpha-\gamma-1} .
\end{aligned}
$$

Denote as follows:

$$
\begin{aligned}
K_{2}:= & \frac{B(\alpha-\delta,(\beta-1)(q-1)+1)}{A \Gamma(\alpha-\gamma)} \\
& \cdot \sum_{i=1}^{m-2} \zeta_{i}\left(\eta_{i}^{\alpha-\delta-1}-\eta_{i}^{\alpha-\delta+(\beta-1)(q-1)}\right) .
\end{aligned}
$$


Since $K_{0}, K_{2}>0$ and $u(t) \geq K_{0}^{q-1} K_{2} t^{\alpha-\gamma-1}$, it is obvious that (71) holds. Therefore, we can see that

$x(t)=I_{0+}^{\gamma} u(t) \geq I_{0+}^{\gamma}\left(K_{0}^{q-1} K_{2} t^{\alpha-\gamma-1}\right)=K_{0}^{q-1} K_{2} \cdot \frac{\Gamma(\alpha-\gamma)}{\Gamma(\alpha)} t^{\alpha-1}$.

The proof is completed.

Remark 15. Theorems 10,12, and 14 show new results that differ from some of the references listed in Section 1. One of these is the result of the existence, uniqueness, and positivity of solutions to $p$-Laplacian fractional differential equations with multipoint boundary conditions where the nonlinear source terms contain a more generalized form of fractional derivatives than those considered in $[16,17,20]$. The other is the possibility of constructing an iterative sequence that converges to a unique solution of a given problem, which is different from $[16,18,19]$. Combining Theorem 14 with Theorems 10 and 12 , the iterative sequence $\left\{x_{n}\right\}_{n=0}^{\infty}$ given by

$$
x_{n}=I_{0+}^{\gamma} u_{n}, n=0,1,2, \cdots,
$$

converges to the exact solution of problem (1), $x$, in $\Omega$, where $\left\{u_{n}\right\}_{n=0}^{\infty}$ is constructed by (68). This gives an opportunity to get the approximate solution, $x_{n}$, for problem (1), although there might be some difficulties in its numerical computation.

3.2. Continuous Dependence of a Solution on Boundary Conditions. In this subsection, the continuous dependence of a unique positive solution $x$ to problem (1) on boundary conditions will be established. To do this, denote a unique solution to the problem

$$
\left\{\begin{array}{l}
D_{0+}^{\beta}\left(\varphi_{p}\left(D_{0+}^{\alpha} x(t)\right)\right)=f\left(t, x(t), D_{0+}^{\gamma} x(t)\right), 0<t<1, \\
D_{0+}^{\gamma} x(0)=0, D_{0+}^{\delta} x(1)=\sum_{i=1}^{m-2} \tilde{\xi}_{i} D_{0+}^{\delta} x\left(\eta_{i}\right), \\
D_{0+}^{\alpha} x(0)=0, \varphi_{p}\left(D_{0+}^{\alpha} x(1)\right)=\sum_{i=1}^{m-2} \tilde{\zeta}_{i} \varphi_{p}\left(D_{0+}^{\alpha} x\left(\eta_{i}\right)\right),
\end{array}\right.
$$

by $\tilde{x}$.

As can be seen above, the positive solution $x$ to problem (1) is given by

$$
x(t)=I_{0+}^{\gamma} u(t), t \in[0,1]
$$

where $u$ is a solution of integral equation (25). Also, the positive solution $\tilde{x}$ to problem (78) is expressed by

$$
\tilde{x}(t)=I_{0+}^{\gamma} \tilde{u}(t), t \in[0,1],
$$

where $\tilde{u}$ is a solution of the integral equation

$$
\tilde{u}(t)=\int_{0}^{1} \tilde{G}(t, s) \phi_{q}\left(\int_{0}^{1} \tilde{H}(s, \tau) f\left(\tau, I_{0+}^{\gamma} \tilde{u}(\tau), \tilde{u}(\tau)\right) d \tau\right) d s,
$$

in which

$$
\begin{aligned}
\tilde{A}= & 1-\sum_{i=1}^{m-2} \tilde{\xi}_{i} \eta_{i}^{\alpha-\delta-1}, \tilde{B}=1-\sum_{i=1}^{m-2} \tilde{\zeta}_{i} \eta_{i}^{\beta-1}, \\
\tilde{G}_{2}(t, s)= & \frac{t^{\alpha-\gamma-1}}{\tilde{A} \Gamma(\alpha-\gamma)}\left[\sum_{s<\eta_{i}} \tilde{\xi}_{i}\left[\eta_{i}^{\alpha-\delta-1}(1-s)^{\alpha-\delta-1}-\left(\eta_{i}-s\right)^{\alpha-\delta-1}\right]\right. \\
& \left.+\sum_{s \geq \eta_{i}} \tilde{\xi}_{i} \eta_{i}^{\alpha-\delta-1}(1-s)^{\alpha-\delta-1}\right], \\
\tilde{H}_{2}(t, s)= & t^{\beta-1}\left[\sum _ { \tilde { B } } \Gamma ( \beta ) \left[\tilde{\zeta}_{i}\left[\eta_{i}^{\beta-1}(1-s)^{\beta-1}-\left(\eta_{i}-s\right)^{\beta-1}\right]\right.\right. \\
& \left.+\sum_{s \geq \eta_{i}} \tilde{\zeta}_{i} \eta_{i}^{\beta-1}(1-s)^{\beta-1}\right], \\
\tilde{G}(t, s)= & G_{1}(t, s)+\tilde{G} \tilde{G}_{2}(t, s), \tilde{H}(t, s)=H_{1}(t, s)+\tilde{H}_{2}(t, s), \\
\widetilde{\bar{M}}= & {[(\alpha-\delta) \tilde{A} \Gamma(\alpha-\gamma)]^{p-1} \tilde{B} \Gamma(\beta), } \\
\tilde{R}{ }_{2}= & \left(M_{a} /\left(\tilde{\bar{M}}-\frac{M_{b}}{\Gamma(\gamma+1)^{p-1}}-M_{c}\right)\right)^{q-1} .
\end{aligned}
$$

Then, the continuous dependence of a unique positive solution to the problem (1) on boundary conditions can be formulated by

$$
\lim _{\substack{\lambda_{\xi} \rightarrow 0 \\ \lambda_{\zeta} \rightarrow 0}} \tilde{x}=x,
$$

where

$$
\begin{aligned}
& \lambda_{\xi}:=\max \left\{\left|\tilde{\xi}_{1}-\xi_{1}\right|,\left|\tilde{\xi}_{2}-\xi_{2}\right|, \cdots,\left|\tilde{\xi}_{m-2}-\xi_{m-2}\right|\right\}, \\
& \lambda_{\zeta}:=\max \left\{\left|\tilde{\zeta}_{1}-\zeta_{1}\right|,\left|\tilde{\zeta}_{2}-\zeta_{2}\right|, \cdots,\left|\tilde{\zeta}_{m-2}-\zeta_{m-2}\right|\right\} .
\end{aligned}
$$

Considering relations (79) and (80), it is sufficient to prove that

$$
\lim _{\substack{\lambda_{\xi} \rightarrow 0 \\ \lambda_{\zeta} \rightarrow 0}} \tilde{u}=u
$$

The solutions of integral equations (25) and (81), $u$ and $\tilde{u}$, uniquely exist in $B_{R_{2}}, B_{\tilde{R}_{2}}$, respectively. Since $A, B$, 
$\bar{M}$, and $R_{2}$ are continuous at $\xi_{1}, \xi_{2}, \cdots, \xi_{m-2}, \zeta_{1}, \zeta_{2}, \cdots, \zeta_{m-2}$, it holds that

$$
\begin{aligned}
& \lim _{\substack{\lambda_{\xi} \rightarrow 0 \\
\lambda_{\zeta} \rightarrow 0}} \tilde{A}=A, \\
& \lim _{\substack{\lambda_{\xi} \rightarrow 0 \\
\lambda_{\zeta} \rightarrow 0}} \tilde{B}=B, \\
& \lim _{\xi \rightarrow 0} \tilde{\bar{M}}=\bar{M}, \\
& \lambda_{\zeta \rightarrow 0} \\
& \lim _{\substack{\lambda_{\xi} \rightarrow 0 \\
\lambda_{\zeta} \rightarrow 0}} \tilde{R}_{2}=R_{2} .
\end{aligned}
$$

Define a function $\bar{G}_{2}(t, s)$ as follows:

$$
\begin{aligned}
& \bar{G}_{2}(t, s) \\
& \quad:=\frac{1}{\Gamma(\alpha-\gamma)}\left\{\begin{array}{l}
t^{\alpha-\delta-1}(1-s)^{\alpha-\delta-1}-(t-s)^{\alpha-\delta-1}, 0 \leq s \leq t \leq 1, \\
t^{\alpha-\delta-1}(1-s)^{\alpha-\delta-1}, 0 \leq t \leq s \leq 1 .
\end{array}\right.
\end{aligned}
$$

Then, it can be easily seen that

$$
G_{2}(t, s)=\frac{1}{A} \sum_{i=1}^{m-2} \xi_{i} \bar{G}_{2}\left(\eta_{i}, s\right) \cdot t^{\alpha-\gamma-1}, \tilde{G}_{2}(t, s)=\frac{1}{\tilde{A}} \sum_{i=1}^{m-2} \tilde{\xi}_{i} \bar{G}_{2}\left(\eta_{i}, s\right) \cdot t^{\alpha-\gamma-1} .
$$

And it can be found in [27] that

$$
H_{2}(t, s)=\frac{1}{B} \sum_{i=1}^{m-2} \zeta_{i} H_{1}\left(\eta_{i}, s\right) \cdot t^{\beta-1}, \tilde{H}_{2}(t, s)=\frac{1}{\tilde{B}} \sum_{i=1}^{m-2} \tilde{\zeta}_{i} H_{1}\left(\eta_{i}, s\right) \cdot t^{\beta-1} .
$$

For a fixed $t$, the functions $\bar{G}_{2}(t, s)$ and $H_{1}(t, s)$ have maximum values at $s=t$, i.e.,

$$
\begin{aligned}
& \max _{s \in[0,1]} \bar{G}_{2}(t, s)=\frac{t^{\alpha-\delta-1}(1-t)^{\alpha-\delta-1}}{\Gamma(\alpha-\gamma)}, \\
& \max _{s \in[0,1]} H_{1}(t, s)=\frac{t^{\beta-1}(1-t)^{\beta-1}}{\Gamma(\beta)} .
\end{aligned}
$$

Lemma 16. Let $y \in C[0,1]$. Then, the following holds:

$$
\begin{aligned}
\forall t \in[0,1],|\tilde{v}(t)-v(t)| \leq & \frac{\|y\|}{\Gamma(\alpha-\gamma)} \sum_{i=1}^{m-2}\left|\frac{\tilde{\xi}_{i}}{\tilde{A}}-\frac{\xi_{i}}{A}\right| \\
& \cdot\left(\eta_{i}^{\alpha-\delta-1}\left(1-\eta_{i}\right)^{\alpha-\delta-1}\right) \cdot t^{\alpha-\gamma-1},
\end{aligned}
$$

where

$$
v(t)=\int_{0}^{1} G(t, s) y(s) d s, \tilde{v}(t)=\int_{0}^{1} \tilde{G}(t, s) y(s) d s .
$$

Proof. Using relation (88), we can see that for any $t \in[0,1]$,

$$
\begin{aligned}
|\tilde{v}(t)-v(t)| & \leq \int_{0}^{1}|\tilde{G}(t, s)-G(t, s)| y(s) d s \\
& \leq\|y\| \int_{0}^{1}\left|\frac{1}{\tilde{A}} \sum_{i=1}^{m-2} \tilde{\xi}_{i} \bar{G}_{2}\left(\eta_{i}, s\right)-\frac{1}{A} \sum_{i=1}^{m-2} \xi_{i} \bar{G}_{2}\left(\eta_{i}, s\right)\right| t^{\alpha-\gamma-1} d s \\
& \leq\|y\| \sum_{i=1}^{m-2}\left|\frac{\tilde{\xi}_{i}}{\tilde{A}}-\frac{\xi_{i}}{A}\right| \int_{0}^{1} \bar{G}_{2}\left(\eta_{i}, s\right) d s \cdot t^{\alpha-\gamma-1} .
\end{aligned}
$$

By applying (90), we obtain

$$
|\tilde{v}(t)-v(t)| \leq \frac{\|y\|}{\Gamma(\alpha-\gamma)} \sum_{i=1}^{m-2}\left|\frac{\tilde{\xi}_{i}}{\tilde{A}}-\frac{\xi_{i}}{A}\right|\left(\eta_{i}^{\alpha-\delta-1}\left(1-\eta_{i}\right)^{\alpha-\delta-1}\right) \cdot t^{\alpha-\gamma-1} .
$$

Lemma 17. Let $z \in C[0,1]$. Then, it holds that

$$
\begin{aligned}
& \forall t \in[0,1],|\tilde{w}(t)-w(t)| \\
& \quad \leq \frac{\|z\| \mid}{\Gamma(\beta)} \sum_{i=1}^{m-2}\left|\frac{\tilde{\zeta}_{i}}{\tilde{B}}-\frac{\zeta_{i}}{B}\right|\left(\eta_{i}^{\beta-1}\left(1-\eta_{i}\right)^{\beta-1}\right) \cdot t^{\beta-1},
\end{aligned}
$$

where

$$
w(t)=\int_{0}^{1} H(t, s) z(s) d s, \tilde{w}(t)=\int_{0}^{1} \tilde{H}(t, s) z(s) d s .
$$

Proof. The proof is similar to Lemma 16, so it is omitted.

Theorem 18. Suppose that all the assumptions of Theorem 10 are satisfied. Then, it holds that

$$
\lim _{\substack{\lambda_{\xi \rightarrow 0} \\ \lambda_{\zeta} \rightarrow 0}} \tilde{u}=u . .
$$

Proof. It can be obtained that for any $t \in[0,1]$,

$$
\begin{aligned}
\mid \tilde{u}(t) & -u(t) \mid \\
\leq & \mid \int_{0}^{1} \tilde{G}(t, s) \varphi_{q}\left(\int_{0}^{1} \tilde{H}(s, \tau) f\left(\tau, I_{0+}^{\gamma} \tilde{u}(\tau), \tilde{u}(\tau)\right) d \tau\right) d s \\
& -\int_{0}^{1} \tilde{G}(t, s) \varphi_{q}\left(\int_{0}^{1} H(s, \tau) f\left(\tau, I_{0+}^{\gamma} u(\tau), u(\tau)\right) d \tau\right) d s \mid \\
& +\mid \int_{0}^{1} \tilde{G}(t, s) \varphi_{q}\left(\int_{0}^{1} H(s, \tau) f\left(\tau, I_{0+}^{\gamma} u(\tau), u(\tau)\right) d \tau\right) d s \\
& \quad-\int_{0}^{1} G(t, s) \varphi_{q}\left(\int_{0}^{1} H(s, \tau) f\left(\tau, I_{0+}^{\gamma} u(\tau), u(\tau)\right) d \tau\right) d s \mid
\end{aligned}
$$




$$
\begin{aligned}
& \leq \int_{0}^{1} \tilde{G}(t, s) \mid \varphi_{q}\left(\int_{0}^{1} \tilde{H}(s, \tau) f\left(\tau, I_{0+}^{\gamma} \tilde{u}(\tau), \tilde{u}(\tau)\right) d \tau\right) \\
& \quad-\varphi_{q}\left(\int_{0}^{1} H(s, \tau) f\left(\tau, I_{0+}^{\gamma} u(\tau), u(\tau)\right) d \tau\right) \mid d s \\
& \quad+\mid \int_{0}^{1}(\tilde{G}(t, s)-G(t, s)) \varphi_{q} \\
& \quad \cdot\left(\int_{0}^{1} H(s, \tau) f\left(\tau, I_{0+}^{\gamma} u(\tau), u(\tau)\right) d \tau\right) d s \mid
\end{aligned}
$$

Lemma 9 indicates that for $u \in B_{R_{2}}, \tilde{u} \in B_{\tilde{R}_{2}}$,

$$
\begin{aligned}
& \int_{0}^{1} H(s, \tau) f\left(\tau, I_{0+}^{\gamma} u(\tau), u(\tau)\right) d \tau \\
& \quad \geq K_{0} s^{\beta-1}, \int_{0}^{1} \tilde{H}(s, \tau) f\left(\tau, I_{0+}^{\gamma} \tilde{u}(\tau), \tilde{u}(\tau)\right) d \tau \geq \tilde{K}_{0} s^{\beta-1},
\end{aligned}
$$

where $\quad \tilde{K}_{0}=\left(\bar{m}^{\sim} \Gamma(\tilde{\mu})\right) /(\tilde{B} \Gamma(\beta+\tilde{\mu})) \sum_{i=1}^{m-2} \tilde{\zeta}_{i}\left(\eta_{i}^{\beta-1}-\eta_{i}^{\beta-1+\tilde{\mu}}\right)$. And by Lemma 11, we know that

$$
\int_{0}^{1} H(s, \tau) f\left(\tau, I_{0+}^{\gamma} u(\tau), u(\tau)\right) d \tau \leq M_{0} .
$$

Considering Lemma 17 and inequality (53), it is easy to see that

$$
\begin{aligned}
& \mid \int_{0}^{1} \tilde{H}(s, \tau) f\left(\tau, I_{0+}^{\gamma} \tilde{u}(\tau), \tilde{u}(\tau)\right) d \tau \\
& \quad-\int_{0}^{1} H(s, \tau) f\left(\tau, I_{0+}^{\gamma} u(\tau), u(\tau)\right) d \tau \mid \\
& \quad \leq \mid \int_{0}^{1} \tilde{H}(s, \tau) f\left(\tau, I_{0+}^{\gamma} \tilde{u}(\tau), \tilde{u}(\tau)\right) d \tau
\end{aligned}
$$

$$
\begin{aligned}
& \quad-\int_{0}^{1} \tilde{H}(s, \tau) f\left(\tau, I_{0+}^{\gamma} u(\tau), u(\tau)\right) d \tau \mid \\
& +\mid \int_{0}^{1} \tilde{H}(s, \tau) f\left(\tau, I_{0+}^{\gamma} u(\tau), u(\tau)\right) d \tau \\
& \quad-\int_{0}^{1} H(s, \tau) f\left(\tau, I_{0+}^{\gamma} u(\tau), u(\tau)\right) d \tau \mid \\
& \leq \int_{0}^{1} \tilde{H}(s, \tau)\left|f\left(\tau, I_{0+}^{\gamma} \tilde{u}(\tau), \tilde{u}(\tau)\right)-f\left(\tau, I_{0+}^{\gamma} u(\tau), u(\tau)\right)\right| d \tau \\
& \quad+\left|\int_{0}^{1}(\tilde{H}(s, \tau)-H(s, \tau)) f\left(\tau, I_{0+}^{\gamma} u(\tau), u(\tau)\right) d \tau\right| \\
& \leq\left(\frac{\max \left\{L_{1}, \tilde{L}_{1}\right\}}{\Gamma(\gamma+1)}+\max \left\{L_{2}, \tilde{L}_{2}\right\}\right)\|\tilde{u}-u\| \int_{0}^{1} \tilde{H}(s, \tau) d \tau \\
& \quad+\frac{C}{\Gamma(\beta)} \sum_{i=1}^{m-2}\left|\frac{\tilde{\zeta}_{i}}{\tilde{B}}-\frac{\zeta_{i}}{B}\right|\left(\eta_{i}^{\beta-1}\left(1-\eta_{i}\right)^{\beta-1}\right) \cdot s^{\beta-1} \\
& \leq\left[\tilde{K}_{1}\left(\frac{\max \left\{L_{1}, \tilde{L}_{1}\right\}}{\Gamma(\gamma+1)}+\max \left\{L_{2}, \tilde{L}_{2}\right\}\right)\|\tilde{u}-u\|\right. \\
& \left.\quad+\frac{C}{\Gamma(\beta)} \sum_{i=1}^{m-2}\left|\frac{\tilde{\zeta}_{i}}{\tilde{B}}-\frac{\zeta_{i}}{B}\right|\left(\eta_{i}^{\beta-1}\left(1-\eta_{i}\right)^{\beta-1}\right)\right] \cdot s^{\beta-1}, \quad(101)
\end{aligned}
$$

where

$$
\tilde{K}_{1}=\frac{1}{\Gamma(\beta+1)}\left[1+\frac{1}{\tilde{B}} \sum_{i=1}^{m-2} \tilde{\zeta}_{i}\left(\eta_{i}^{\beta-1}-\eta_{i}^{\beta}\right)\right],
$$

$$
\text { and } C=\max _{\substack{t \in[0,1] \\ x \in\left[0, R_{1}\right] \\ y \in\left[0, R_{2}\right]}}|f(t, x, y)| \text {. }
$$
that

Therefore, combining Lemma 16 with $1<q<2$, it follows that

$$
\begin{aligned}
|\tilde{u}(t)-u(t)| \leq & \frac{(q-1)}{\min \left\{K_{0}^{2-q}, \tilde{K}_{0}^{2-q}\right\}}\left[\tilde{K}_{1}\left(\frac{\max \left\{L_{1}, \tilde{L}_{1}\right\}}{\Gamma(\gamma+1)}+\max \left\{L_{2}, \tilde{L}_{2}\right\}\right)\|\tilde{u}-u\|+\frac{C}{\Gamma(\beta)} \sum_{i=1}^{m-2}\left|\frac{\tilde{\zeta}_{i}}{\tilde{B}}-\frac{\zeta_{i}}{B}\right|\left(\eta_{i}^{\beta-1}\left(1-\eta_{i}\right)^{\beta-1}\right)\right] \\
& \cdot \int_{0}^{1} \tilde{G}_{*}(s, s) s^{(\beta-1)(q-1)} d s+\frac{M_{0}}{\Gamma(\alpha-\gamma)} \sum_{i=1}^{m-2}\left|\frac{\tilde{\zeta}_{i}}{\tilde{A}}-\frac{\xi_{i}}{A}\right|\left(\eta_{i}^{\alpha-\delta-1}\left(1-\eta_{i}\right)^{\alpha-\delta-1}\right) \\
\leq & \frac{(q-1)\left[\tilde{K}_{1}\left(\left(\left(\max \left\{L_{1}, \tilde{L}_{1}\right\}\right) /(\Gamma(\gamma+1))\right)+\max \left\{L_{2}, \tilde{L}_{2}\right\}\right)\|\tilde{u}-u\|+(C / \Gamma(\beta)) \sum_{i=1}^{m-2}\left|\left(\tilde{\zeta}_{i} / \tilde{B}^{\prime}\right)-\left(\zeta_{i} / B\right)\right|\left(\eta_{i}^{\beta-1}\left(1-\eta_{i}\right)^{\beta-1}\right)\right]}{\tilde{A}(\alpha-\delta) \Gamma(\alpha-\gamma) \min \left\{K_{0}^{2-q}, \tilde{K}_{0}^{2-q}\right\}} \\
& +\frac{M_{0}}{\Gamma(\alpha-\gamma)} \sum_{i=1}^{m-2}\left|\tilde{\xi}_{i}\right| \frac{\xi_{i}}{\tilde{A}}-\left(\eta_{i}^{\alpha-\delta-1}\left(1-\eta_{i}\right)^{\alpha-\delta-1}\right) \cdot
\end{aligned}
$$


Since

$$
\frac{(q-1) K_{1}}{A(\alpha-\delta) \Gamma(\alpha-\gamma) K_{0}^{2-q}}\left(\frac{L_{1}}{\Gamma(\gamma+1)}+L_{2}\right)<1
$$

and $A, B, K_{0}, K_{1}, L_{1}, L_{2}$ are continuous at $\xi_{1}, \xi_{2}, \cdots, \xi_{m-2}, \zeta_{1}$, $\zeta_{2}, \cdots, \zeta_{m-2}$, we can see that for sufficient small $\lambda_{\xi}$ and $\lambda_{\zeta}$,

$$
\begin{aligned}
\Lambda_{1}:= & \frac{(q-1) \tilde{K}_{1}}{\tilde{A}(\alpha-\delta) \Gamma(\alpha-\gamma) \min \left\{K_{0}^{2-q}, \tilde{K}_{0}^{2-q}\right\}} \\
& \cdot\left(\frac{\max \left\{L_{1}, \tilde{L}_{1}\right\}}{\Gamma(\gamma+1)}+\max \left\{L_{2}, \tilde{L}_{2}\right\}\right)<1 .
\end{aligned}
$$

So, putting

$$
\begin{aligned}
& \Lambda_{2}:=\frac{(q-1) C}{\tilde{A}(\alpha-\delta) \Gamma(\beta) \Gamma(\alpha-\gamma) \min \left\{K_{0}^{2-q}, \tilde{K}_{0}^{2-q}\right\}}, \\
& \Lambda_{3}:=\frac{M_{0}}{\Gamma(\alpha-\gamma)},
\end{aligned}
$$

$$
\|\tilde{u}-u\| \leq \frac{\Lambda_{2} \sum_{i=1}^{m-2}\left|\left(\tilde{\zeta}_{i} / \tilde{B}\right)-\left(\zeta_{i} / B\right)\right|\left(\eta_{i}^{\beta-1}\left(1-\eta_{i}\right)^{\beta-1}\right)+\Lambda_{3} \sum_{i=1}^{m-2}\left|\left(\tilde{\xi}_{i} / \tilde{A}\right)-\left(\xi_{i} / A\right)\right|\left(\eta_{i}^{\alpha-\delta-1}\left(1-\eta_{i}\right)^{\alpha-\delta-1}\right)}{1-\Lambda_{1}} .
$$

Finally, the fact that

$$
\lim _{\substack{\lambda_{\xi} \rightarrow 0 \\ \lambda_{\zeta} \rightarrow 0}} \frac{\tilde{\xi}_{i}}{\tilde{A}}=\frac{\xi_{i}}{A}, \lim _{\substack{\lambda_{\xi} \rightarrow 0 \\ \lambda_{\zeta} \rightarrow 0}} \frac{\tilde{\zeta}_{i}}{\tilde{B}}=\frac{\zeta_{i}}{B}, i=1,2, \cdots, m-2
$$

proves the conclusion of this theorem.

Theorem 19. Suppose that all the assumptions of Theorem 12 hold. Then, it is satisfied that

$$
\lim _{\substack{\lambda_{\xi} \rightarrow 0 \\ \lambda_{\zeta} \rightarrow 0}} \tilde{u}=u
$$

Proof. Similar to Theorem 18, we have that for any $t \in[0,1]$,

$$
\begin{aligned}
|\tilde{u}(t)-u(t)| \leq & \int_{0}^{1} \tilde{G}(t, s) \mid \varphi_{q}\left(\int_{0}^{1} \tilde{H}(s, \tau) f\left(\tau, I_{0+}^{\gamma} \tilde{u}(\tau), \tilde{u}(\tau)\right) d \tau\right) \\
& -\varphi_{q}\left(\int_{0}^{1} H(s, \tau) f\left(\tau, I_{0+}^{\gamma} u(\tau), u(\tau)\right) d \tau\right) \mid d s \\
& +\mid \int_{0}^{1}(\tilde{G}(t, s)-G(t, s)) \varphi_{q} \\
& \cdot\left(\int_{0}^{1} H(s, \tau) f\left(\tau, I_{0+}^{\gamma} u(\tau), u(\tau)\right) d \tau\right) d s \mid
\end{aligned}
$$

By Lemma 11, we know that for $u \in B_{R_{2}}, \tilde{u} \in B_{\tilde{R}_{2}}$,

$$
\begin{aligned}
& \int_{0}^{1} H(s, \tau) f\left(\tau, I_{0+}^{\gamma} u(\tau), u(\tau)\right) d \tau \leq M_{0}, \\
& \int_{0}^{1} H(s, \tau) f\left(\tau, I_{0+}^{\gamma} \tilde{u}(\tau), \tilde{u}(\tau)\right) d \tau \leq \tilde{M}_{0},
\end{aligned}
$$

where

$$
\tilde{M}_{0}=\frac{M_{a}+\left(\left(M_{b} /\left(\Gamma(\gamma+1)^{p-1}\right)\right)+M_{c}\right) \tilde{R}_{2}^{p-1}}{\tilde{B} \Gamma(\beta)} .
$$

From Lemma 17 and the inequality (53), we obtain

$$
\begin{aligned}
& \left|\int_{0}^{1} \tilde{H}(s, \tau) f\left(\tau, I_{0+}^{\gamma} \tilde{u}(\tau), \tilde{u}(\tau)\right) d \tau-\int_{0}^{1} H(s, \tau) f\left(\tau, I_{0+}^{\gamma} u(\tau), u(\tau)\right) d \tau\right| \\
& \leq \int_{0}^{1} \tilde{H}(s, \tau)\left|f\left(\tau, I_{0+}^{\gamma} \tilde{u}(\tau), \tilde{u}(\tau)\right)-f\left(\tau, I_{0+}^{\gamma} u(\tau), u(\tau)\right)\right| d \tau \\
& \quad+\left|\int_{0}^{1}(\tilde{H}(s, \tau)-H(s, \tau)) f\left(\tau, I_{0+}^{\gamma} u(\tau), u(\tau)\right) d \tau\right| \\
& \leq \quad\left(\frac{\max \left\{L_{1}, \tilde{L}_{1}\right\}}{\Gamma(\gamma+1)}+\max \left\{L_{2}, \tilde{L}_{2}\right\}\right)\|\tilde{u}-u\| \\
& \quad \cdot \int_{0}^{1} \tilde{H}_{*}(s, s) d \tau+\frac{C}{\Gamma(\beta)} \sum_{i=1}^{m-2}\left|\tilde{\zeta}_{i}\right| \frac{\zeta_{i}}{\tilde{B}} \mid\left(\eta_{i}^{\beta-1}\left(1-\eta_{i}\right)^{\beta-1}\right) \cdot s^{\beta-1} \\
& \leq\left(\frac{\max \left\{L_{1}, \tilde{L}_{1}\right\}}{\Gamma(\gamma+1)}+\max \left\{L_{2}, \tilde{L}_{2}\right\}\right) \frac{\|\tilde{u}-u\|}{\tilde{B} \Gamma(\beta+1)} \\
& \quad+\frac{C}{\Gamma(\beta)} \sum_{i=1}^{m-2}\left|\frac{\tilde{\zeta}_{i}}{\tilde{B}}-\frac{\zeta_{i}}{B}\right|\left(\eta_{i}^{\beta-1}\left(1-\eta_{i}\right)^{\beta-1}\right),
\end{aligned}
$$

where

$$
C=\max _{\substack{t \in[0,1] \\ x \in\left[0, R_{1}\right] \\ y \in\left[0, R_{2}\right]}}|f(t, x, y)| .
$$


Since $q>2$, using Lemma 16, we can get

$$
\begin{aligned}
|\tilde{u}(t)-u(t)| \leq & {\left[\left(\frac{\max \left\{L_{1}, \tilde{L}_{1}\right\}}{\Gamma(\gamma+1)}+\max \left\{L_{2}, \tilde{L}_{2}\right\}\right) \frac{\|\tilde{u}-u\|}{\tilde{B} \Gamma(\beta+1)}+\frac{C}{\Gamma(\beta)} \sum_{i=1}^{m-2}\left|\left(\tilde{\zeta}_{i} / \tilde{B}\right)-\left(\zeta_{i} / B\right)\right|\left(\eta_{i}^{\beta-1}\left(1-\eta_{i}\right)^{\beta-1}\right)\right] } \\
& \cdot(q-1) \max \left\{M_{0}^{q-2}, \tilde{M}_{0}^{q-2}\right\} \int_{0}^{1} \tilde{G}_{*}(s, s) d s+\frac{M_{0}}{\Gamma(\alpha-\gamma)} \sum_{i=1}^{m-2}\left|\frac{\tilde{\xi}_{i}}{\tilde{A}}-\frac{\xi_{i}}{A}\right|\left(\eta_{i}^{\alpha-\delta-1}\left(1-\eta_{i}\right)^{\alpha-\delta-1}\right) \\
\leq & \frac{\left[\left(\left(\max \left\{L_{1}, \tilde{L}_{1}\right\} / \Gamma(\gamma+1)\right)+\max \left\{L_{2}, \tilde{L}_{2}\right\}\right)(\|\tilde{u}-u\| /(\tilde{B} \Gamma(\beta+1)))+(C / \Gamma(\beta)) \sum_{i=1}^{m-2}\left|\tilde{\zeta}_{i} / \tilde{B}_{-}-\zeta_{i} / B\right|\left(\eta_{i}^{\beta-1}\left(1-\eta_{i}\right)^{\beta-1}\right)\right]}{\tilde{A}(\alpha-\delta) \Gamma(\alpha-\gamma)} \\
& \cdot(q-1) \max \left\{M_{0}^{q-2}, \tilde{M}_{0}^{q-2}\right\}+\frac{M_{0}}{\Gamma(\alpha-\gamma)} \sum_{i=1}^{m-2}\left|\frac{\tilde{\xi}_{i}}{\tilde{A}}-\frac{\xi_{i}}{A}\right|\left(\eta_{i}^{\alpha-\delta-1}\left(1-\eta_{i}\right)^{\alpha-\delta-1}\right) .
\end{aligned}
$$

From the condition

$$
\begin{gathered}
\frac{(q-1) M_{0}^{q-2}}{(\alpha-\delta) A B \Gamma(\alpha-\gamma) \Gamma(\beta+1)}\left(\frac{L_{1}}{\Gamma(\gamma+1)}+L_{2}\right) \\
=\frac{(q-1) R_{2}^{2-p}}{\beta \bar{M}}\left(\frac{L_{1}}{\Gamma(\gamma+1)}+L_{2}\right)<1,
\end{gathered}
$$

it holds that for sufficiently small $\lambda_{\xi}$ and $\lambda_{\zeta}$,

$$
\begin{aligned}
\Lambda_{4}:= & \frac{(q-1) \max \left\{M_{0}^{q-2}, \tilde{M}_{0}^{q-2}\right\}}{\tilde{A}(\alpha-\delta) \Gamma(\alpha-\gamma) \tilde{B} \Gamma(\beta+1)} \\
& \cdot\left(\frac{\max \left\{L_{1}, \tilde{L}_{1}\right\}}{\Gamma(\gamma+1)}+\max \left\{L_{2}, \tilde{L}_{2}\right\}\right)<1 .
\end{aligned}
$$

Denote as follows:

$$
\Lambda_{5}:=\frac{(q-1) \max \left\{M_{0}^{q-2}, \tilde{M}_{0}^{q-2}\right\} C}{\tilde{A}(\alpha-\delta) \Gamma(\beta) \Gamma(\alpha-\gamma)}, \Lambda_{6}:=\frac{M_{0}}{\Gamma(\alpha-\gamma)}
$$

$$
\|\tilde{u}-u\| \leq \frac{\Lambda_{5} \sum_{i=1}^{m-2}\left|\left(\tilde{\zeta}_{i} / \tilde{B}\right)-\left(\zeta_{i} / B\right)\right|\left(\eta_{i}^{\beta-1}\left(1-\eta_{i}\right)^{\beta-1}\right)+\Lambda_{6} \sum_{i=1}^{m-2}\left|\left(\tilde{\xi}_{i} / \tilde{A}\right)-\left(\xi_{i} / A\right)\right|\left(\eta_{i}^{\alpha-\delta-1}\left(1-\eta_{i}\right)^{\alpha-\delta-1}\right)}{1-\Lambda_{4}} .
$$

Since

$$
\lim _{\substack{\lambda_{\xi} \rightarrow 0 \\ \lambda_{\zeta} \rightarrow 0}} \frac{\tilde{\xi}_{i}}{\tilde{A}}=\frac{\xi_{i}}{A}, \lim _{\substack{\lambda_{\xi} \rightarrow 0 \\ \lambda_{\zeta} \rightarrow 0}} \frac{\tilde{\zeta}_{i}}{\tilde{B}}=\frac{\zeta_{i}}{B}, i=1,2, \cdots, m-2,
$$

the proof is completed.

Remark 20. It follows from Theorems 18 and 20 that $\lim \tilde{x}=x$. Therefore, it follows that the unique positive $\lambda_{\xi} \rightarrow 0$

$\lambda_{\zeta}^{\zeta} \rightarrow 0$

solution to problem (1) is continuously dependent on the perturbations with respect to the coefficients in $m$-point boundary conditions. This is a new result different from the achievements of Dishlieva [21] and Li et al. [22] who studied the continuous dependency of solutions on initial conditions, barrier curves, and source terms in differential equations but not on boundary conditions.

\section{Examples}

Example 21. Consider the following boundary value problem:

$$
\left\{\begin{array}{l}
D_{0+}^{1.5}\left(\varphi_{3}\left(D_{0+}^{1.75} x(t)\right)\right)=10 \sqrt{t}+0.2 x(t)+0.1\left[D_{0+}^{0.2} x(t)\right]^{2}, 0<t<1, \\
D_{0+}^{0.2} x(0)=0, D_{0+}^{0.4} x(1)=0.3 D_{0+}^{0.4} x(0.3)+0.2 D_{0+}^{0.4} x(0.7), \\
D_{0+}^{1.75} x(0)=0, \varphi_{3}\left(D_{0+}^{1.75} x(1)\right)=0.2 \varphi_{3}\left(D_{0+}^{1.75} x(0.3)\right)+0.5 \varphi_{3}\left(D_{0+}^{1.75} x(0.7)\right) .
\end{array}\right.
$$


Problem (121) can be regarded as the boundary value problem (1) where $f(t, x, y)=10 \sqrt{t}+0.2 x+0.1 y^{2}, p=3$, $\alpha=1.75, \beta=1.5, \gamma=0.2, \delta=0.4, \eta_{1}=0.3, \eta_{2}=0.7, \xi_{1}=0.3$, $\xi_{2}=0.2, \zeta_{1}=0.2$, and $\zeta_{2}=0.5$. Then, we have

$$
\begin{aligned}
q & =1.5<2, \\
A & =0.626632 \cdots, \\
B & =0.472125 \cdots, \\
\bar{M} & =0.236575 \cdots
\end{aligned}
$$

Since $x \leq 4+\left(x^{2} / 16\right)$, denoting $a(t)=10.8, b(t)=0.0125$, and $c(t)=0.1$ yields that

$$
\begin{aligned}
M_{a} & =10.8, \\
M_{b} & =0.0125, \\
M_{c} & =0.1, \\
f(t, x, y) & <a(t)+b(t) x^{p-1}+c(t) y^{p-1} .
\end{aligned}
$$

Putting $\bar{m}=10, \mu=1.5$, we find that

$$
f(t, x, y) \geq \bar{m} t^{\mu-1} .
$$

$R_{2}, R_{1}$ can be calculated as

$$
\begin{aligned}
& R_{2}=9.41849 \cdots, \\
& R_{1}=\frac{R_{2}}{\Gamma(\gamma+1)}=10.2579 \cdots .
\end{aligned}
$$

Also, put $L_{1}=0.2, L_{2}=0.1 \cdot 2$, and $R_{2}=1.8837 \cdots$. Then, we can see that for any $t \in[0,1]$ and any $\left(x_{1}, y_{1}\right),\left(x_{2}, y_{2}\right) \in$ $\left[0, R_{1}\right] \times\left[0, R_{2}\right]$,

$$
\begin{aligned}
\left|f\left(t, x_{1}, y_{1}\right)-f\left(t, x_{1}, y_{2}\right)\right| & \leq 0.2\left|x_{1}-x_{2}\right|+0.1\left|y_{1}^{2}-y_{2}^{2}\right| \\
& \leq L_{1}\left|x_{1}-x_{2}\right|+L_{2}\left|y_{1}-y_{2}\right| .
\end{aligned}
$$

A simple calculation provides that

$$
\begin{aligned}
K_{0} & =2.48598 \cdots, \\
K_{1} & =1.07439 \cdots, \\
K & =0.952215 \cdots .
\end{aligned}
$$

By Theorems 10 and $14, K<1$ shows that fractional boundary value problem (121) has a unique positive solution in $I_{0+}^{\gamma}\left(B_{R_{2}}\right)$. Besides, Theorem 18 shows that the unique positive solution to the problem (121) is continuously dependent on its boundary conditions.
Example 22. Consider the boundary value problem

$$
\left\{\begin{array}{l}
D_{0+}^{1.5}\left(\varphi_{1.5}\left(D_{0+}^{1.75} x(t)\right)\right)=0.1 t+0.1[x(t)]^{2}+0.1 D_{0+}^{0.2} x(t), 0<t<1, \\
D_{0+}^{0.2} x(0)=0, D_{0+}^{0.4} x(1)=0.3 D_{0+}^{0.4} x(0.3)+0.2 D_{0+}^{0.4} x(0.7), \\
D_{0+}^{1.75} x(0)=0, \varphi_{1.5}\left(D_{0+}^{1.75} x(1)\right)=0.2 \varphi_{1.5}\left(D_{0+}^{1.75} x(0.3)\right)+0.5 \varphi_{1.5}\left(D_{0+}^{1.75} x(0.7)\right) .
\end{array}\right.
$$

Problem (128) can be regarded as the boundary value problem (1) where $f(t, x, y)=0.1 t+0.1 x^{2}+0.1 y, p=1.5$, $\alpha=1.75, \beta=1.5, \gamma=0.2, \delta=0.4, \eta_{1}=0.3, \eta_{2}=0.7, \quad \xi_{1}=0.3$, $\xi_{2}=0.2, \zeta_{1}=0.2$, and $\zeta_{2}=0.5$. Then, we can get

$$
\begin{aligned}
q & =3>2, \\
A & =0.626632 \cdots, \\
B & =0.472125 \cdots, \\
\bar{M} & =0.362822 \cdots,
\end{aligned}
$$

Denote $a(t)=0.1, b(t)=0.1, c(t)=0.1$ and we have

$$
\begin{aligned}
& M_{a}=0.1, \\
& M_{b}=0.1, \\
& M_{c}=0.1, \\
& R_{2}=0.398248 \cdots, \\
& R_{1}=0.433742 \cdots .
\end{aligned}
$$
$\left[0, R_{2}\right]$,

Also, it holds that for any $(t, x, y) \in[0,1] \times\left[0, R_{1}\right] \times$

$$
f(t, x, y)=0.1 t+0.1 x^{2}+0.1 y<0.1+0.1 \sqrt{x}+0.1 \sqrt{y} .
$$

Since $\left|f\left(t, x_{1}, y_{1}\right)-f\left(t, x_{2}, y_{2}\right)\right| \leq 0.1\left|x_{1}^{2}-x_{2}^{2}\right|+0.1 \mid y_{1}$ $-y_{2}\left|\leq 0.1 \cdot 2 R_{1}\right| x_{1}-x_{2}|+0.1 \cdot| y_{1}-y_{2} \mid$ for any $t \in[0,1]$ and any $\left(x_{1}, y_{1}\right),\left(x_{2}, y_{2}\right) \in\left[0, R_{1}\right] \times\left[0, R_{2}\right]$, we can put

$$
\begin{aligned}
& L_{1}=0.0867483 \cdots, \\
& L_{2}=0.1 .
\end{aligned}
$$

By simple calculation, we have

$$
K=0.45102 \cdots
$$

Since $K<1$, using Theorems 12 and 14, boundary value problem (128) has a unique positive solution in $I_{0+}^{\gamma}\left(B_{R_{2}}\right)$. Also, by employing Theorem 20, the continuous dependence of the unique positive solution to problem (128) on its boundary conditions is proved.

Comparing the problems (121) and (128) considered in Examples 22 and 23, respectively, there exists two main 
differences between them. One is the value of $p$. If $p=2$, it is obvious that $\varphi_{p}(s)=s$. As can be seen in the inequalities (23) and (24), some properties of $\varphi_{p}(\cdot)$ depend on whether the value of $p$ is greater than 2 or not. So, Examples 22 and 23 demonstrated the existence, the uniqueness, the positivity, and the continuous dependency on four-point boundary conditions of the solutions to the problems (121) and (128) in the cases of $p>2$ and $1<p<2$ by using Theorems 10 and 18 and Theorems 12 and 20, respectively. The other difference lies in the nonlinear source term $f(t, x, y)$. The source term in problem (121) was nonlinear to the fractional derivative of the unknown function, whereas, in problem (128), the nonlinearity appeared for the unknown function itself. However, similar techniques were applied to these two nonlinear source terms in the problems (121) and (128) to illustrate our main results.

\section{Conclusion}

In this paper, we have proved the existence, the uniqueness, the positivity, and the continuous dependency on multipoint boundary conditions of solutions to $m$-point boundary value problems for nonlinear multiterm fractional differential equations with $p$-Laplacian operator by employing the Banach contraction mapping principle. For the application of this study, two examples have been illustrated.

\section{Data Availability}

No data were used to support this study.

\section{Conflicts of Interest}

There is no competing interest among the authors regarding the publication of the article.

\section{Authors' Contributions}

All authors carried out the proof and conceived of the study. All authors read and approved the final manuscript.

\section{References}

[1] A. A. Kilbas, H. M. Srivastava, and J. J. Trujillo, Theory and Applications of Fractional Differential Equations, Elsevier, Amsterdam, The Netherlands, 2006.

[2] I. Podlubny, Fractional Differential Equations, Academic Press, San Diego, USA, 1999.

[3] L. S. Leibenson, "General problem of the movement of a compressible fluid in a porous medium," Izvestiya Akademii Nauk Kirgizskoı̆ SSR, vol. 9, pp. 7-10, 1945.

[4] J. R. Esteban and J. L. Vazquez, "On the equation of turbulent filtration in one- dimensional porous media," Nonlinear Analysis: Theory, Methods \& Applications, vol. 10, no. 11, pp. 13031325, 1986.

[5] M. A. Herrero and J. L. Vazquez, "On the propagation properties of a nonlinear degenerate parabolic equation," Communications in Partial Differential Equations, vol. 7, no. 12, pp. 1381-1402, 1982.
[6] L. E. Bobisud, "Steady state turbulent flow with reaction," Rocky Mountain Journal of Mathematics, vol. 21, no. 3, pp. 993-1007, 1991.

[7] J. J. Zhang, W. B. Liu, T. Y. Chen, and H. X. Zhang, "Solvability of $p$-Laplace equations subject to three-point boundary value problems," Applied Mathematics and Computation, vol. 179, no. 2, pp. 688-695, 2006.

[8] Y. Guo, Y. Ji, and X. Liu, "Multiple positive solutions for some multi-point boundary value problems with $p$-Laplacian," Journal of Computational and Applied Mathematics, vol. 216, no. 1, pp. 144-156, 2008.

[9] Y. Zhang, "Existence and multiplicity results for a class of generalized one-dimensional p-Laplacian problem," Nonlinear Analysis: Theory, Methods \& Applications, vol. 72, no. 2, pp. 748-756, 2010.

[10] J. Zhao and W. Ge, "Multiple positive solutions for some multi-point boundary value problems with p-Laplacian," Computers \& Mathematics with Applications, vol. 58, no. 6, pp. 1103-1112, 2009.

[11] Z. Lv, "Existence results for $m$-point boundary value problems of nonlinear fractional differential equations with $p$-Laplacian operator," Advances in Difference Equations, vol. 2014, Article ID 69, 2014.

[12] Y. Li and A. Qi, "Positive solutions for multi-point boundary value problems of fractional differential equations with $p$ Laplacian," Mathematical Methods in the Applied Sciences, vol. 39, no. 6, pp. 1425-1434, 2016.

[13] X. Dong, Z. Bai, and S. Zhang, "Positive solutions to boundary value problems of $p$-Laplacian with fractional derivative," Boundary Value Problems, vol. 2017, Article ID 5, 2017.

[14] K. Jong, H. Choi, K. Jang, and S. Pak, "Existence and uniqueness results for a class of singular fractional boundary value problems with the-Laplacian operator via the upper and lower solutions approach," Journal of Function Spaces, vol. 2020, Article ID 2930892, 15 pages, 2020.

[15] N. Nyamoradi and S. Tersian, "Existence of solutions for nonlinear fractional order $p$-Laplacian differential equations via critical point theory," Fractional Calculus \& Applied Analysis, vol. 22, no. 4, pp. 945-967, 2019.

[16] T. Chen, W. Liu, and Z. Hu, "A boundary value problem for fractional differential equation with $p$-Laplacian operator at resonance," Nonlinear Analysis: Theory, Methods \& Applications, vol. 75, pp. 3210-3217, 2012.

[17] X. Liu, M. Jia, and W. Ge, "The method of lower and upper solutions for mixed fractional four-point boundary value problem with p-Laplacian operator," Applied Mathematics Letters, vol. 65, pp. 56-62, 2017.

[18] W. Jiang, "Solvability of fractional differential equations with p-Laplacian at resonance," Applied Mathematics and Computation, vol. 260, pp. 48-56, 2015.

[19] Y. Su, Q. Li, and X. Liu, "Existence criteria for positive solutions of $p$-Laplacian fractional differential equations with derivative terms," Advances in Difference Equations, vol. 2013, Article ID 119, 2013.

[20] Y. Su, Y. Yun, D. Wang, and W. Hu, "Existence of solutions to nonlinear $p$-Laplacian fractional differential equations with higher-order derivative terms," Electronic Journal of Differential Equations, vol. 2018, article 105, 2018.

[21] K. G. Dishlieva, "Continuous dependence of the solutions of impulsive differential equations on the initial conditions and 
barrier curves," Acta Mathematica Scientia, vol. 32B, no. 3, pp. 1035-1052, 2012.

[22] Z. Li, Y. Liu, and M. Yamamoto, "Initial-boundary value problems for multi-term time-fractional diffusion equations with positive constant coefficients," Applied Mathematics and Computation, vol. 257, pp. 381-397, 2015.

[23] S. G. Samko, A. A. Kilbas, and O. I. Marichev, Fractional Integrals and Derivatives: Theory and Applications, Gordon and Breach, Yverdon, Switzerland, 1993.

[24] Z. Bai and H. Lu, "Positive solutions for boundary value problem of nonlinear fractional differential equation," Journal of Mathematical Analysis and Applications, vol. 311, no. 2, pp. 495-505, 2005.

[25] K. Jong, "Existence and uniqueness of positive solutions of a kind of multi-point boundary value problems for nonlinear fractional differential equations with $p$-Laplacian Operator," Mediterranean Journal of Mathematics, vol. 15, no. 3, 2018.

[26] X. Liu, M. Jia, and X. Xiang, "On the solvabilityof a fractionaldifferential equation model involving the $p$-Laplacian operator," Computers \& Mathematics with Applications, vol. 64, no. 10, pp. 3267-3275, 2012.

[27] K. Jong, H. Choi, and Y. Ri, "Existence of positive solutions of a class of multi-point boundary value problems for $p$-Laplacian fractional differential equations with singular source terms," Communications in Nonlinear Science and Numerical Simulation, vol. 72, pp. 272-281, 2019. 